\title{
Improving Energy Efficiency in the Production Floor Using SoA-Based Monitoring Techniques
}

\author{
Daniel Cachapa ${ }^{1,2}$, Robert Harrison ${ }^{1}$, and Armando Colombo ${ }^{2}$ \\ ${ }^{1}$ Wolfson School of Mechanical and Manufacturing Engineering, Loughborough University, \\ Loughborough, LE11 3TU, United Kingdom \\ \{D.Cachapa-Vieira, R.Harrison\} @lboro.ac.uk \\ ${ }^{2}$ Schneider Electric Automation GmbH, Industry Business Unit, Steinheimer Str. 117, \\ 63500 Seligenstadt, Germany \\ \{Daniel.Cachapa, Armando.Colombo\}@de.schneider-electric.com
}

\begin{abstract}
Modern manufacturing systems are struggling to remain competitive under the pressures of and increasingly demanding society. The advent of SoA-based production systems is presented as the solution to facing those difficulties. In order to support this new production paradigm, new tools have to be developed for the benefit of the production engineers of who will be implementing and deploying the SoA-based factory floor. This entails the development of Smart Devices, which while being autonomous are able to communicate and cooperate in an open and standardized way with other participants in the production system. These capabilities can be exploited in order to implement a next-generation production monitoring system that is not only able to monitor production status, but also energy usage, eventually leading to much greener and more efficient factories.
\end{abstract}

Keywords: SoA, Web Services, Production Monitoring, Simulation.

\section{Introduction}

Modern industrial manufacturing is facing pressure from all directions: governments want greener and safer products, while costumers demand quality, customization and lower prices. In order to face these challenges in an age of technological boom, companies are turning more and more to machines to accomplish the tasks previously made by human.

The field of factory automation has been a pioneer in this area and has evolved much in the past few years as machines get ever more sophisticated and efficient. Nevertheless, these changes have also raised the complexities involved in maintaining and controlling them. Implementing a modern factory floor is an incredibly expensive and time-consuming labour as production specifications must be transformed into the machine code connecting all of the devices together. Likewise, a monitoring framework must also be implemented so that production engineers can have a real-time view of the machine's status, production flow, energy usage, stock management, as well as other essential production indexes. 
It is expected that the event-based, high-level and decoupled nature of SoA-enabled production devices will allow for easier integration, configuration and maintenance of factory monitoring systems, while at the same time improving the performance and capabilities over traditional systems. The study of monitoring and energy efficiency issues in SoA-based factory automation relies not only on following current state-ofthe-art in the relevant areas, but also on the building of prototype engineering solutions that demonstrate the proposed methodologies. Those solutions should be engineered based on existing use-cases that represent current industrial needs.

\section{Contribution to Technological Innovation}

The flexible production line requires the development of modular devices which can be plugged into the production line and participate in a cooperative network configured to accomplish a given task. The devices themselves should be autonomous in the sense that they don't depend directly on any of the other participants in the network to achieve their full functionality, which means that the devices are individually programmed and tested before reaching the production line.

This entails:

- Developing building blocks - Intelligent functions embedded into devices (profiles, HMI, tools, web, agents).

- Making the blocks work together - Design of networked autonomous and fault adaptive systems (protocols, robust, security).

- Assuring a common objective - Concepts, methods and tools for building robust, reconfigurable intelligent systems and guarantee expected overall system behaviour.

Also important is that the devices are able to communicate in a standards-based manner with the other participants in the network. Standardization guarantees better flexibility for the system builder in mixing and matching parts from different suppliers, creating a fairer market for device builders and thereby reducing costs through the power of healthy competition in the marketplace.

The requirements described above are embodied in the tenants of SoA as it describes a standards-based network of autonomous participants which work together in order to reach a common goal. Specifically, the use of SoA and Web Service technology from the enterprise level all the way down to the device level yields the following benefits:

- Easy adoption: it is possible to deploy the technology incrementally either by gradually replacing components, or using middleware solutions in older equipment (Priyantha, Kansal, Goraczko, \& Zhao, 2008).

- Easy integration: made possible by the standards-based nature of SoA and WS technology.

- Easy to develop new applications: SoA and Web Services are at the heart of new programming paradigms heavily endorsed by influential software companies such as Microsoft ${ }^{\circledR}$ and Sun ${ }^{\circledR}$.

- Reduced time for setup: made possible by the high-level nature of Web Services, and facilities such as discovery and eventing. 
Bringing SoA into the production line requires a careful design in order to keep a clean architecture with clear hierarchical separation between different levels and usage patterns. The implementation must account for the requirements of the current production runs, as well as being ready to quickly incorporate changes in the production process, while at the same time supporting use cases that can range from production control to production monitoring, to business process management (Karnouskos, Baecker, de Sousa, \& Spieß, 2007).

With energy prices are on the rise, and environmental concerns coming from both consumers and governmental agencies, the industry is concerning itself greatly with energy savings on the shop floor, which not only have the potential of yielding savings directly on the energy bills and environmental taxes, but also having an impact on sales as the marketing department is allowed to stick a "green" label on the enduser packaging.

The research presented here aims to develop a SoA-based framework for decoupled factory devices, and use it to perform monitoring of production data, with a particular emphasis on energy usage, in order to research viable energy management strategies for the factories of the future.

\section{State-of-the-Art / Related Literature}

Many SoA-based frameworks have been proposed, such as in (Colombo, 2008), where the author separates the production system into three hierarchies: the embedded components (TEC), composed into embedded machines (TEM), which themselves are arranged into embedded production systems (TES). In (Lastra, 2004), the author presents a similar approach dubbed Actor-based Assembly Systems in which simple SoA-based devices with limited functionality are composed together to build complex production systems. In \{Document Not In Library\} the authors present a similar framework of SoA-based devices using the OPC Unified Architecture. These frameworks have many things in common, which represent the central tenets of the SoAbased production system: a society of autonomous devices which cooperate in order to achieve the functionality required for the production objectives.

The research presented here builds upon the same basic procedures and techniques in order to build a fully functional production system. Much of the work follows the groundwork laid down in the SIRENA (Jammes \& Smit, 2005) and in the SOCRADES (de Souza et al., 2008) projects, as well as the RI-MACS project \{Document Not In Library\}. It is also closely related to the objectives put forward by the CONET \{Document Not In Library\} research project.

The developments and tools developed in support of the research are mainly supported upon the Devices Profile for Web Services (DPWS) communication stack (Jammes, Mensch, \& Smit, 2005) under the development umbrella of the SoA4D group \{Document Not In Library\}, and the Delmia Automation CAD suite \{Document Not In Library\} as a simulation/visualization tool. The results of the early experiments with these sets of tools are described in (Cachapa, Colombo, Feike, \& Bepperling, 2007) and (Leitão et al., 2009). 


\section{Research Contribution and Innovation}

The research plan for the work presented here is separated in two stages: the development of a SoA-based framework for factory devices, and the leveraging of that framework in order to perform event-based monitoring of production and energy data.

The first and most essential step in the creation of a test production line based on the SoA paradigm is the availability of "smart devices". The concept of a Smart Device is used to describe a device that is designed from the ground up to be autonomous and cooperative. Smart devices integrate the kinematics; the behaviour, which is exposed to the outside as services; and, in the case of a virtual device, the geometry, or physical dimensions of the device it represents. Smart Devices can thus be brought into the production line, connected to the network, and have their full functionality immediately available for the controller to exploit with minimal configuration. The procedure of developing a virtual smart device in the Delmia Automation environment with a DPWS-based service interface which is identical to the one available from the corresponding real device is described in (Cachapa, Colombo, Feike, \& Bepperling, 2007).

The Smart Devices feature fixed input and output ports at its physical boundaries, so that different devices can be connected at their ports in order to build a continuous sequence of machines that can transport work pieces inside the production cell. The ports themselves are intimately connected to the device's internal functionality and exposed as individual services, meaning that the physical composition of devices is also reflected in the functional composition of the respective services associated to those ports. Two or more devices can be composed in this manner in order to form composed devices, which abstract the interactions between the individual devices and whose resulting characteristics may be different from the simple sum of the capabilities and limitations of each component. The composed devices can be themselves composed into new composed devices of an even higher hierarchy.

By linking and composing smart devices together, a full, SoA-based production system can be assembled. This technique was demonstrated in (Leitão et al., 2009) where a virtual SoA-based production cell, shown in Fig. 1a) was built according to an existing physical cell, shown in Fig.1b), and used to aid the development of the production controller.

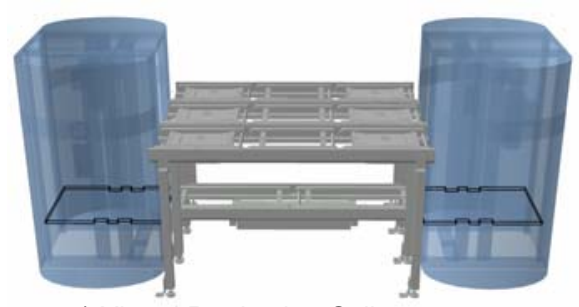

a) Virtual Production Cell

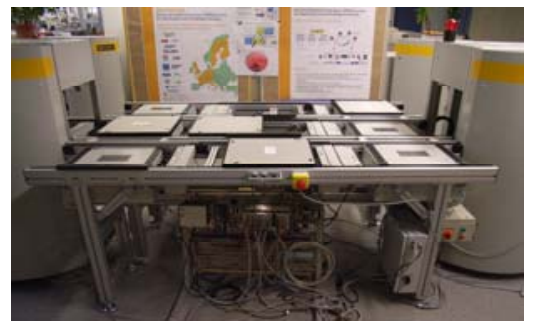

b) Real Production Cell

Fig. 1. Virtual and real representations of a SoA-based production cell 
Since all participants in the production line share the same network and high-level functionality, and the components in both the virtual and the real environments appear in the network as autonomous devices, there is no difference in functionality between them from the controller's point of view, which allows replacing some of the virtual components for real ones for testing purposes. The outcome is an open architecture where all the participants can communicate freely according to their function, as seen in Fig. 2.

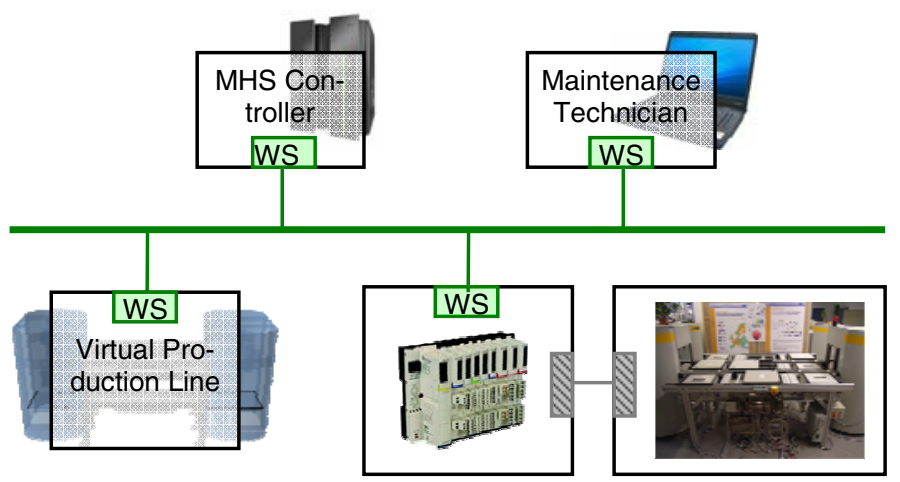

Fig. 2. Virtual SoA-based production system with hardware in the middle

Thanks to the high-level nature of the services provided by the smart devices, the device operations have a one-to-one mapping between both the real and virtual worlds, meaning that as long as all the devices are accessible in the network, the same controller is able to perform the same set of operations in the virtual and real worlds without the need for any specific platform dependencies. Fig. 3 shows an example of this mapping during a transfer operation from one smart device to another.

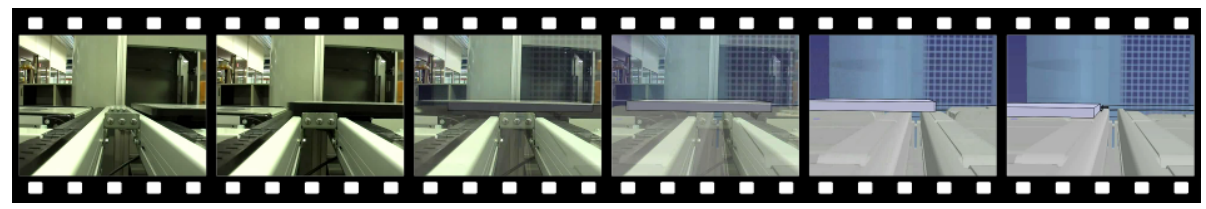

Fig. 3. Mapping operations in the real world to the virtual world using SoA-based real and virtual smart devices

This technique allows the production engineer to validate the production cell's layout and process control on the virtual production floor, and later replace the virtual devices for real ones as necessary for testing until the control software is running the full, physical, production cell. Similarly, the installation of a new device can be tested offline by first connecting it to the virtual model, and replacing it with the real device only after the new configuration has been validated. 


\subsection{Event-Based Monitoring of Production and Energy Data}

The initial steps towards a SoA-based production monitoring system for factory automation begin with a study of existing systems and a gathering of the requirements for such a system, as well as a wish list for improvements. The results of this study were as expected: monitoring systems are proprietary, in-house solutions, and are based on a patchwork of barely compatible and sometimes outdated technologies.

This is the outcome of the systems having been built many years ago to face the growing pains of production automation, and then growing organically to face the ever increasing complexity of today's factories. The end result is a complex system layout featuring multiple closed networks interconnected by data exchangers, bridges and various middleware solutions.

The same SoA-based platform which is useful for aiding development of production systems could be used for performing real-time production monitoring. The event-based nature of the Smart Devices, coupled with other state-of-the-art technologies, such as RFID, allows for efficient tracking of materials, resources and operations over the production lifecycle. This would make it possible to use a single, open and standards compliant network throughout the entire factory floor, which would facilitate integration of new devices in already existing facilities.

Furthermore, the same system can be used to plug in to the various energy consumers participating in the production system, such as the facilities, lighting, heating, and others. Having detailed real-time and historical energy data will go a long way towards finding energy inefficiencies in the production process and researching ways of optimizing or eliminating them.

\section{Discussion of Results and Critical View}

The results achieved so far demonstrate that a SoA-based engineering tool can support the production engineer by greatly simplifying the process of production line layout and configuration. The simulation capabilities offered by the tool are indispensible for quick prototyping and testing of the capabilities, performance and throughput of the production process.

Furthermore, both the high-level nature the Web Service-based components and the generic support of the virtual 3D platform enable the quick development of new components and their adaptation into an existing production environment, where they can be thoroughly tested before deployment.

After deployment, the same technologies can serve as the base to a next-generation production monitoring system, replacing the current proprietary ones which face enormous limitations due to their complexity and forced support for legacy technologies.

The open and standard nature of the technologies and protocols used in the communication between the different devices enable a future where factories don't have to rely on a limited set of vendors for all of their production or control devices, but instead, benefit from an ecosystem where competing companies which all develop their products targeting the same standards, giving rise to a modular factory floor where the different machines are mixed-and-matched according to their quality, capabilities and price. 
That's not to say that a new system would solve all the problems overnight. Initially, the SoA-based production monitoring system would either function parallel to the existing systems, or legacy technology would have to be adapted through the use of middleware software.

\section{Conclusions and Further Work}

It is unquestionable that the current tools for production engineering aren't capable of supporting the advent of SoA in the factory floor. For this reason, new tools and techniques will have to be developed.

The research presented here lays the groundwork for developing SoA-based virtual production lines based on proven industrial standards. These tools are an essential platform for conducting further research in the fields of production control and monitoring.

The first glimpses on the current state of production monitoring in use today show that there is much that can be improved. The event-based and open nature of the SoAbased production system makes it ideal for tackling the problem of monitoring the factory floor. It is hoped that the research into these issues culminates in a standard, extensible set of guidelines for production monitoring systems which follow closely current industrial requirements.

Acknowledgments. The authors would like to thank the European Commission and the partners of the EU IST FP6 project "Service-Oriented Cross-layer infrastructure for Distributed smart Embedded devices" (SOCRADES) and the EC ICT FP7 NoE project "Cooperating Objects" (CONET) for their support. Further recognition is due to the brilliant engineers at the Ford Motor Company in Dunton UK.

\section{References}

1. Priyantha, N., Kansal, A., Goraczko, M., Zhao, F.: Tiny Web Services: Design and Implementation of Interoperable and Evolvable Sensor Networks. In: Proceedings of the 6th ACM Conference on Embedded Network Sensor Systems, pp. 253-266. ACM, New York (2008)

2. Karnouskos, S., Baecker, O., de Sousa, L., Spieß, P.: Integration of SOA-ready Networked Embedded Devices in Enterprise Systems via a Cross-Layered Web Service Infrastructure. In: Proceedings of the 12th IEEE Conference on Emerging Technologies and Factory Automation, pp. 293-300. IEEE Computer Society, Los Alamitos (2007)

3. Colombo, A.: Integration of Web-Services and Agent Technology: A Service-Oriented Architecture-based Automation Framework. In: Workshop Agenten in der Automatisierungstechnik (2008)

4. Lastra, J.: Reference Mechatronic Architecture for Actor-Based Assembly Systems. Doctoral thesis, Tampere University of Technology (2004)

5. Jammes, F., Smit, H.: Service-oriented Architectures for Devices - the SIRENA view. In: 3rd IEEE International Conference on Industrial Informatics, pp. 140-147. IEEE Computer Society, Los Alamitos (2005) 
6. de Souza, L., Spiess, P., Guinard, D., Kohler, M., Karnouskos, S., Savio, D.: SOCRADES: A Web Service Based Shop Floor Integration Infrastructure. In: Floerkemeier, C., Langheinrich, M., Fleisch, E., Mattern, F., Sarma, S.E. (eds.) IOT 2008. LNCS, vol. 4952, pp. 50-67. Springer, Heidelberg (2008)

7. Jammes, F., Mensch, A., Smit, H.: Service-oriented Device Communications Using the Devices Profile for Web Services. In: Proceedings of the 3rd International Workshop on Middleware for Pervasive and Ad-hoc Computing, pp. 1-8. ACM, New York (2005)

8. Cachapa, D., Colombo, A., Feike, M., Bepperling, A.: An Approach for Integrating Real and Virtual Production Automation Devices Applying the Service-oriented Architecture Paradigm. In: IEEE Conference on Emerging Technologies and Factory Automation, pp. 309-314. IEEE Computer Society, Los Alamitos (2007)

9. Leitão, P., Mendes, J., Bepperling, A., Cachapa, D., Colombo, A., Restivo, F.: Engineering Tools for the Integration of Service-oriented Production Systems. In: 13th IFAC Symposium on Information Control Problems in Manufacturing (2009) 